\title{
Estimates on the number of orbits of the Dyck shift
}

Fahad Alsharari ${ }^{1 *}$ (D) Mohd Salmi Md Noorani ${ }^{1}$ and Habibulla Akhadkulov ${ }^{1,2}$

\section{"Correspondence:}

fahd331@gmail.com

'School of Mathematical Sciences,

Faculty of Science and Technology,

Universiti Kebangsaan Malaysia,

Bangi, 43600, Malaysia

Full list of author information is

available at the end of the article

\begin{abstract}
In this paper, we get crucial estimates of fundamental sums that involve the number of closed orbits of the Dyck shift. These estimates are given as the prime orbit theorem, Mertens' orbit theorem, Meissel's orbit theorem and Dirichlet series. Different and more direct methods are used in the proofs without any complicated theoretical discussions.
\end{abstract}

Keywords: the Dyck shift; closed orbits; dynamical analogs

\section{Introduction}

In number theory, the prime-counting function that gives the number of primes less than or equal to $x$, for any real number $x$ is denoted by $\pi(x)$. The prime number theorem (PNT) is the statement

$$
\pi(x) \sim x / \log (x)
$$

This means that

$$
\lim _{x \rightarrow \infty} \frac{\pi(x)}{x / \log (x)}=1
$$

However, the following asymptotic formula for primes $p$ :

$$
\prod_{p \leq x}\left(1-\frac{1}{p}\right) \sim \frac{e^{-\gamma}}{\log x}
$$

where $\gamma=0.5772 \ldots$ is Euler's constant and $x \in \mathbf{R}$, is known as Mertens' theorem of analytic number theory. The logarithmic equivalent of Mertens' theorem is

$$
\sum_{p \leq x} \frac{1}{p}=\log \log x+B_{1}+O\left(\frac{1}{\log x}\right),
$$

and Mertens' constant $B_{1}=0.2614972128 \ldots$, where $f=O(g)$ means that $f(x)$ is bounded with respect to $g(x)$ for all sufficiently large $x$.

(c) 2015 Alsharari et al. This article is distributed under the terms of the Creative Commons Attribution 4.0 International License (http://creativecommons.org/licenses/by/4.0/), which permits unrestricted use, distribution, and reproduction in any medium, provided you give appropriate credit to the original author(s) and the source, provide a link to the Creative Commons license, and indicate if changes were made. 
Also, as pointed out by Lindqvist and Peetre [1], Meissel considered the sum

$$
\sum_{p} \frac{1}{p(\ln p)^{a}} \approx \frac{1}{a}+M
$$

where $M=\lim _{x \rightarrow \infty} \sum_{p \leq x} \frac{1}{p}-\ln (\ln x) \approx 0.2614972128 \ldots$

A Dirichlet series is the complex function defined on $\{z \in \mathbf{C}: \operatorname{Re}(z)>1\}$ by the series

$$
d(z)=\sum_{n=1}^{\infty} \frac{a_{n}}{n^{z}}
$$

where $z$ and $a_{n}$ are complex numbers, $n=1,2,3, \ldots$ If $a_{n}=1$ for all $n$ then the Dirichlet series

$$
d(z)=\sum_{n=1}^{\infty} \frac{1}{n^{z}},
$$

becomes the Riemann zeta function [2].

Let $X$ be a non-empty set and $T: X \rightarrow X$ be a map. The pair $(X, T)$ is said to be a dynamical system. Many dynamical questions involve counting the number of closed orbits or the periodic points under iteration of a map. A closed (periodic) orbit $\tau$ of length $|\tau|=n$ for a continuous map $T: X \rightarrow X$ is a set of the form $\left\{x, T x, T^{2} x, \ldots, T^{n-1} x\right\} \subset X$ where $T^{n} x=x$ for some $x \in X$ and $n \geq 1$.

Let $T: X \rightarrow X$ be a map, and define

$$
\begin{aligned}
& \mathcal{L}_{T}(n)=\left\{x \in X:\left|\left\{T^{k}(x)\right\}\right|_{k \in \mathbf{N}}=n\right\}, \\
& \mathcal{F}_{T}(n)=\left\{x \in X: T^{n}(x)=x\right\} \text { and } \\
& \mathcal{O}_{T}(n)=\{\tau: \tau \text { is a closed orbit of } T \text { of length }|\tau|=n\},
\end{aligned}
$$

which are the set of points of least period $n$ under $T$, the set of points of period $n$ under $T$, and the set of closed orbits of length $n$ under $T$, respectively. The Möbius inversion formula [3] is defined as follows. If $f, g: \mathbf{N} \rightarrow \mathbf{C}$ are two arithmetic functions satisfying

$$
g(n)=\sum_{d \mid n} f(d) \quad \forall n
$$

then

$$
f(n)=\sum_{d \mid n} \mu\left(\frac{n}{d}\right) g(d) \quad \forall n
$$

where $\mu(n)$ is the Möbius function. Let

$$
L_{T}(n)=\left|\mathcal{L}_{T}(n)\right|, \quad F_{T}(n)=\left|\mathcal{F}_{T}(n)\right| \quad \text { and } \quad O_{T}(n)=\left|\mathcal{O}_{T}(n)\right| .
$$

It follows that

$$
O_{T}(n)=L_{T}(n) / n
$$


and

$$
F_{T}(n)=\sum_{d \mid n} L_{T}(d)
$$

Consequently

$$
F_{T}(n)=\sum_{d \mid n} d O_{T}(d)
$$

and hence, by the Möbius inversion formula,

$$
O_{T}(n)=\frac{1}{n} \sum_{d \mid n} \mu\left(\frac{n}{d}\right) F_{T}(d)
$$

Inspired by the functions of an algebraic variety over a finite field we are led to obtain a kind of analogs between number theory and dynamical systems. Following the analogy between closed orbits and prime numbers, the asymptotic behavior of expressions like

$$
\pi_{T}(N)=\sum_{n \leq N} O_{T}(n)
$$

may be viewed as a dynamical analog of the prime number theorem and a dynamical ana$\log$ of Mertens' theorem concerns asymptotic estimates for expressions like

$$
\mathcal{M}_{T}(N)=\sum_{n \leq N} \frac{O_{T}(n)}{e^{h n}}
$$

where $h$ denotes the topological entropy of the map $T$. Moreover, the dynamical analog of Meissel's theorem is given as an infinite sum of the form

$$
\sum_{n=1}^{\infty} \frac{O_{T}(n)}{n^{a} e^{h n}}
$$

as $a \rightarrow 0^{+}$.

Furthermore, the dynamical Dirichlet series associated to the map $T: X \rightarrow X$ is the formal series of the form

$$
d_{T}(z)=\sum_{n=1}^{\infty} \frac{O_{T}(n)}{n^{z}},
$$

which alternatively can be expressed as

$$
d_{T}(z)=\frac{1}{\zeta(z+1)} \sum_{n=1}^{\infty} \frac{F_{T}(n)}{n^{z+1}},
$$

where $\zeta(z)$ is the dynamical zeta function given by

$$
\zeta_{T}(z)=\exp \sum_{n=1}^{\infty} \frac{F_{T}(n)}{n} z^{n} .
$$


The dynamical zeta function is introduced by [4] and based also on an analogy with the number theory zeta functions attached to a function field over a finite field and is one of the tools used in studying orbit-growth properties of maps.

Parry in [5] initiated a line of research which uses ideas and techniques of analytic number theory to attack problems of this nature. When $X$ has a metric structure with respect to which $T$ is hyperbolic or a shift of finite type, results of Parry and Pollicott [6] and Noorani [7] have shown a similar analogy between the number of closed orbits and the prime number theorem. It has been shown that

$$
\pi_{T}(N) \sim \frac{e^{h(N+1)}}{N\left(e^{h}-1\right)} .
$$

Sharp in [8] also obtained an analogy between the number of closed orbits and Mertens' theorem for hyperbolic maps as follows:

$$
\mathcal{M}_{T}(N) \sim \log N+C_{1}
$$

for some constant $C_{1}$.

Several orbit-counting results on the asymptotic behavior of both (5) and (6) for other maps like quasihyperbolic toral automorphism (ergodic but not hyperbolic), can be found for example in [9-11] and [12].

In this paper, analogs between the number of closed orbits of a shift of infinite type called the Dyck shift and (1), (2), (3), and (4) have been obtained. Instead of using the zeta function approach to prove our results, different and more direct methods are used without any complicated theoretical discussion.

Authors in [13], have obtained analogs between the number of closed orbits of the Dyck shift and only (1) and (2). However, later authors have explored a minor mistake in the proof of the first theorem. In this note, we improve the minor mistake detected in the proof of the first theorem, which yields a more correct estimate. We also obtain sharper estimates for the second theorem in [13].

\section{The Dyck shift}

A complete introduction to the theory of symbolic dynamics can be found in [14]. Let $\mathcal{A}$ be a finite alphabet. On $\mathcal{A}^{\mathbf{Z}}$ there acts the shift that sends the point $\left(x_{i}\right)_{i \in \mathbf{Z}} \in \mathcal{A}^{\mathbf{Z}}$ into the point $\left(x_{i+1}\right)_{i \in \mathbf{Z}} \in \mathcal{A}^{\mathbf{Z}}$. The dynamical systems that are given by the closed shift invariant subsets of $\mathcal{A}^{\mathbf{Z}}$, with the restriction of the shift acting on them, are called subshifts. These are studied in symbolic dynamics. An element of $\mathcal{A}^{n}$ will be called a word, or a block of length $n$. A word of length 0 is called an empty word and denoted by $\varepsilon$. The set of all finite words with letters taken from $\mathcal{A}$ is the set $\mathcal{A}^{*}=\bigcup_{n=0}^{\infty} \mathcal{A}^{n}$. A word is called admissible for the subshift $X \subset \mathcal{A}^{\mathbf{Z}}$ if it appears somewhere in a point of $X$. Let $X \subset \mathcal{A}^{\mathbf{Z}}$ and let $\mathcal{B}_{n}(X)$ denote the set of all admissible words of length $n$ in $X$. Then the language of $X$ is the collection $\mathcal{B}(X)=\bigcup_{n=0}^{\infty} \mathcal{B}_{n}(X)$. The topological entropy of a subshift $X \subset \mathcal{A}^{\mathrm{Z}}$ is given by

$$
h(X)=\lim _{n \rightarrow \infty} \frac{1}{n} \log \left|\mathcal{B}_{n}(X)\right|
$$

Let $\mathcal{A}=\left\{\ell_{1}, \ell_{2}, \ldots, \ell_{M}, r_{1}, r_{2}, \ldots, r_{M}\right\}$. The alphabet consists of $M$ pairs of matching left and right delimiters or symbols. Let $\mathcal{M}$ be a monoid (with zero) with generators $\ell_{i}, r_{i}$, 
$1 \leq i \leq M$, and 1 . The relations on the monoid are

$$
\begin{aligned}
& \ell_{i} \circ r_{j}=1 \quad \text { if } i=j 1 \leq i, j \leq M, \\
& \ell_{i} \circ r_{j}=0 \quad \text { if } i \neq j 1 \leq i, j \leq M, \\
& \alpha \circ 1=1 \circ \alpha=\alpha, \quad \alpha \in \mathcal{A} \cup\{1\}, \\
& \alpha \circ 0=0 \circ \alpha=0, \quad \alpha \in \mathcal{A} \cup\{1\}, \\
& 0 \circ 0=0 .
\end{aligned}
$$

We use a mapping $\operatorname{red}(): \mathcal{A}^{*} \rightarrow \mathcal{M}$ such that for

$$
\begin{aligned}
& \omega=\omega_{1} \omega_{2} \cdots \omega_{n} \in \mathcal{A}^{*} \quad(n \geq 1), \\
& \operatorname{red}(\omega)=\omega_{1} \circ \omega_{2} \circ \cdots \circ \omega_{n} \quad \text { and } \quad \operatorname{red}(\varepsilon)=1 .
\end{aligned}
$$

The Dyck shift $\mathrm{D}_{M}[15]$ is defined by

$$
\mathrm{D}_{M}=\left\{x \in \mathcal{A}^{\mathrm{Z}}: \text { if } i \leq j \text {, then } \operatorname{red}\left(x_{[i, j)}\right) \neq 0\right\}
$$

where $x_{[i, j)}=x_{i} x_{i+1} \cdots x_{j-1}$.

When $M=1, \mathrm{D}_{1}$ is the full shift on two symbols; we will tacitly assume that $M \geq 2$. The topological entropy of the Dyck shift $\mathrm{D}_{M}$ is already computed as $\log (M+1)$ in [15].

The number of points [16] in the Dyck shift $\mathrm{D}_{M}$ having period $n$ is given by

$$
F(n)= \begin{cases}2\left\{(M+1)^{n}-\sum_{i=0}^{\frac{n}{2}}\left(\begin{array}{c}
n \\
i
\end{array}\right) M^{i}\right\}+\left(\begin{array}{c}
n \\
n
\end{array}\right) M^{\frac{n}{2}} & \text { if } n \text { is even; } \\
2\left\{(M+1)^{n}-\sum_{i=0}^{(n-1) / 2}\left(\begin{array}{c}
n \\
i
\end{array}\right) M^{i}\right\} & \text { if } n \text { is odd. }\end{cases}
$$

\section{Counting closed orbits}

In this section, we first give a lower bound and an upper bound for the number of periodic points of the Dyck shift and then apply such a result to obtain asymptotic formulas for the dynamical analogs of the prime number theorem, Merten's theorem, Meissel's theorem, and Dirichlet series.

Lemma 3.1 There exist constants $0<c_{1}<1$ and $c_{2}>1$ such that the following inequality holds for all $n \geq 1$ :

$$
c_{1}(M+1)^{n} \leq F(n) \leq c_{2}(M+1)^{n},
$$

where $F(n)$ denotes the number of periodic points in the Dyck shift.

Proof Assume that $n$ is even. The case $n$ is odd can be proved analogously. Note then first that

$$
\sum_{i=0}^{\frac{n}{2}}\left(\begin{array}{c}
n \\
i
\end{array}\right) M^{i}<(M+1)^{n} \text { and }\left(\begin{array}{c}
n \\
\frac{n}{2}
\end{array}\right) M^{\frac{n}{2}}<(M+1)^{n} .
$$


Therefore, we obtain

$$
\begin{aligned}
F(n) & =2(M+1)^{n}\left\{1-\frac{\sum_{i=0}^{\frac{n}{2}}\left(\begin{array}{c}
n \\
i
\end{array}\right) M^{i}}{(M+1)^{n}}+\frac{\left(\begin{array}{c}
n \\
\frac{n}{2}
\end{array}\right) M^{\frac{n}{2}}}{(M+1)^{n}}\right\} \\
& <3(M+1)^{n} \quad \text { for all } n \geq 1 .
\end{aligned}
$$

Also

$$
\begin{aligned}
F(n) & \geq(M+1)^{n}-\sum_{i=0}^{\frac{n}{2}}\left(\begin{array}{c}
n \\
i
\end{array}\right) M^{i}+\left(\begin{array}{c}
n \\
\frac{n}{2}
\end{array}\right) M^{\frac{n}{2}} \\
& \geq(M+1)^{n}\left[1-\frac{\sum_{i=0}^{\frac{n}{2}}\left(\begin{array}{c}
n \\
i
\end{array}\right) M^{i}}{(M+1)^{n}}\right] .
\end{aligned}
$$

Estimating the term $\left[1-\frac{\sum_{i=0}^{\frac{n}{2}}\left(\begin{array}{c}n \\ i\end{array}\right) M^{i}}{(M+1)^{n}}\right]$ using the binomial theorem we obtain

$$
\begin{aligned}
\frac{\sum_{i=0}^{\frac{n}{2}}\left(\begin{array}{c}
n \\
i
\end{array}\right) M^{i}}{(M+1)^{n}} & =\frac{\sum_{i=0}^{\frac{n}{2}}\left(\begin{array}{c}
n \\
i
\end{array}\right) M^{i}}{\sum_{i=0}^{\frac{n}{2}-1}\left(\begin{array}{c}
n \\
i
\end{array}\right) M^{i}+\left(\begin{array}{c}
n \\
\frac{n}{2}
\end{array}\right) M^{\frac{n}{2}}+\sum_{i=\frac{n}{2}+1}^{n}\left(\begin{array}{c}
n \\
i
\end{array}\right) M^{i}} \\
& \leq \frac{\sum_{i=0}^{\frac{n}{2}}\left(\begin{array}{c}
n \\
i
\end{array}\right) M^{i}}{\sum_{i=0}^{\frac{n}{2}-1}\left(\begin{array}{c}
n \\
i
\end{array}\right) M^{i}+\sum_{i=\frac{n}{2}+1}^{n}\left(\begin{array}{c}
n \\
i
\end{array}\right) M^{i}}
\end{aligned}
$$

We set

$$
\begin{aligned}
& \mathrm{A}_{n}:=\sum_{i=0}^{\frac{n}{2}-1}\left(\begin{array}{l}
n \\
i
\end{array}\right) M^{i}=\sum_{i=0}^{\frac{n}{2}-1}\left(\begin{array}{c}
n \\
\frac{n}{2}-i-1
\end{array}\right) M^{\frac{n}{2}-i-1}, \\
& \mathrm{~B}_{n}:=\sum_{i=n+1}^{n}\left(\begin{array}{l}
n \\
i
\end{array}\right) M^{i}=\sum_{i=0}^{\frac{n}{2}-1}\left(\begin{array}{c}
n \\
\frac{n}{2}+i+1
\end{array}\right) M^{\frac{n}{2}+i+1},
\end{aligned}
$$

using the definition of the binomial coefficient it is easy to prove that

$$
\left(\begin{array}{c}
n \\
\frac{n}{2}-i-1
\end{array}\right)=\left(\begin{array}{c}
n \\
\frac{n}{2}+i+1
\end{array}\right) \text { for any } 0 \leq i \leq \frac{n}{2}-1
$$

also

$$
M^{\frac{n}{2}+i+1} \geq M^{2}\left(M^{\frac{n}{2}-i-1}\right) \quad \text { for any } 0 \leq i \leq \frac{n}{2}-1
$$

and utilizing (9) and (10) we obtain

$$
\mathrm{B}_{n} \geq M^{2}\left(\mathrm{~A}_{n}\right)
$$

Applying inequality (11) to (8), we get

$$
\frac{\sum_{i=0}^{\frac{n}{2}}\left(\begin{array}{c}
n \\
i
\end{array}\right) M^{i}}{(M+1)^{n}} \leq \frac{\mathrm{A}_{n}}{\mathrm{~A}_{n}+\mathrm{B}_{n}} \leq \frac{\mathrm{A}_{n}}{\mathrm{~A}_{n}+M^{2}\left(\mathrm{~A}_{n}\right)}=\frac{1}{1+M^{2}},
$$


hence, inequality (7) implies that

$$
F(n) \geq(M+1)^{n}\left(1-\frac{1}{1+M^{2}}\right)=\frac{M^{2}}{1+M^{2}}(M+1)^{n} .
$$

Thus, if we take $c_{1}=\frac{M^{2}}{1+M^{2}}$ and $c_{2}=3$, then we are done.

Theorem 3.2 (Prime orbit theorem) Let $\sigma: \mathrm{D}_{M} \rightarrow \mathrm{D}_{M}$ be the Dyck shift. Let $\pi(N)=$ $\sum_{n \leq N} O_{\sigma}(n)$ be the number of closed orbits of $\sigma$ not exceeding $N$. Then there exist constants $C_{1}$ and $C_{2}$ such that

$$
C_{1} \frac{(M+1)^{N}}{N} \leq \sum_{n \leq N} O_{\sigma}(n) \leq C_{2} \frac{(M+1)^{N}}{N}
$$

Proof For the sake of notation let us denote $A=M+1$. First of all by using Lemma 3.1, we would like to show that

$$
c_{3} F(N) \leq \sum_{n \leq N} F(n) \leq c_{4} F(N) .
$$

Indeed,

$$
\sum_{n \leq N} F(n)=F(N) \sum_{n \leq N} \frac{F(n)}{F(N)} \leq \frac{c_{2}}{c_{1}} F(N) \sum_{n=0}^{N-1} \frac{1}{A^{n}} \leq \frac{c_{2} A}{c_{1}(A-1)} F(N) .
$$

Similarly

$$
\sum_{n \leq N} F(n)=F(N) \sum_{n \leq N} \frac{F(n)}{F(N)} \geq \frac{c_{1}}{c_{2}} F(N) .
$$

Now using (12) we prove the main theorem. Applying the Möbius inversion formula to the functions $F(n)$ and $O(n)$, where $O(n)$ is the number of closed orbits with length $n$, we have

$$
\begin{aligned}
\sum_{n \leq N} O_{\sigma}(n) & =\sum_{n \leq N} \frac{1}{n} \sum_{d \mid n} \mu\left(\frac{n}{d}\right) F(d) \\
& =\sum_{n \leq N} \frac{1}{n} \sum_{d \mid n, d<n} \mu\left(\frac{n}{d}\right) F(d)+\sum_{n \leq N} \frac{1}{n} F(n) .
\end{aligned}
$$

Subtracting the dominant terms, we obtain

$$
\begin{aligned}
\left|\sum_{n \leq N} O_{\sigma}(n)-\sum_{n \leq N} \frac{1}{n} F(n)\right| & \leq\left|\sum_{n \leq N} \frac{1}{n} \sum_{d \mid n, d<n} \mu\left(\frac{n}{d}\right) F(d)\right| \leq \sum_{n \leq N} \sum_{d \leq\left\lfloor\frac{n}{2}\right\rfloor} F(d) \\
& \leq \sum_{n \leq N} F\left(\frac{n}{2}\right) \leq \frac{c_{2} A}{c_{1}(A-1)}\left(F\left(\frac{N}{2}\right)\right) .
\end{aligned}
$$


To estimate the dominant terms, let $K(N)=\left\lfloor N^{\frac{1}{4}}\right\rfloor$. Then

$$
\begin{aligned}
\left|\sum_{n \leq N} \frac{1}{n} F(n)-\sum_{N-K(N) \leq n \leq N} \frac{1}{n} F(n)\right| & \leq \sum_{n \leq N-K(N)} F(n) \\
& \leq \frac{c_{2} A}{c_{1}(A-1)}(F(N-K(N))) .
\end{aligned}
$$

Utilizing Lemma 3.1 we have

$$
\begin{aligned}
\sum_{N-K(N) \leq n \leq N} \frac{1}{n} F(n) & =\frac{F(N)}{N}\left[\sum_{r=0}^{K(N)}\left(\frac{F(N-r)}{F(N)} \cdot \frac{N}{N-r}\right)\right] \\
& \leq \frac{c_{2} A^{N}}{N}\left[\sum_{r=0}^{K(N)}\left(\frac{c_{2}}{c_{1} A^{r}} \cdot\left(\sum_{n=0}^{\infty}\left(\frac{r}{N}\right)^{n}\right)\right]\right] \\
& =\frac{c_{3} A^{N}}{N}\left[\sum_{r=0}^{K(N)} \frac{1}{A^{r}}+\sum_{r=0}^{K(N)} \sum_{j=1}^{\infty}\left(\frac{r}{N}\right)^{j}\right] \\
& \leq \frac{c_{3} A^{N}}{N}\left[\frac{A}{A-1}+c_{4}\left(\frac{(K(N))^{2}}{N}\right)\right] .
\end{aligned}
$$

Therefore, summarizing (13), (14), and (15) and using $K(N) \leq N^{\frac{1}{4}}$ we get

$$
\sum_{n \leq N} O_{\sigma}(n) \leq c_{5} \frac{A^{N}}{N}\left(1+\frac{N}{A^{\left[\frac{N}{2}\right]}}+\frac{N}{A^{\left[\frac{N}{4}\right]}}+\frac{1}{\sqrt{N}}\right) \leq c_{6} \frac{A^{N}}{N} .
$$

Hence we have obtained an upper bound. To obtain the lower bound, first we estimate the following sum for $n \geq 8$ :

$$
\begin{aligned}
\sum_{d \mid n} \mu\left(\frac{n}{d}\right) F(d) & =\sum_{d \mid n, d<n} \mu\left(\frac{n}{d}\right) F(d)+\mu(1) F(n) \\
& =F(n)+\sum_{d \leq\left\lfloor\frac{n}{2}\right\rfloor} \mu\left(\frac{n}{d}\right) F(d) \geq F(n)-\sum_{d \leq\left\lfloor\frac{n}{2}\right\rfloor} F(d) \\
& \geq c_{1} A^{n}-c_{2}\left(A+A^{2}+\cdots+A^{\left\lfloor\frac{n}{2}\right\rfloor}\right) \\
& =c_{1} A^{n}-c_{2}\left(\frac{A\left(A^{\left\lfloor\frac{n}{2}\right\rfloor+1}-1\right)}{A-1}\right) \\
& =c_{1} A^{n}\left(1-\frac{c_{2}}{c_{1}}\left(\frac{A\left(A^{\left\lfloor\frac{n}{2}\right\rfloor+1}-1\right)}{A^{n}(A-1)}\right)\right) \geq \frac{c_{1}}{2} A^{n}
\end{aligned}
$$

for all $n \geq 8$. Therefore, using (17) we obtain

$$
\begin{aligned}
\sum_{n \leq N} O_{\sigma}(n) & =\sum_{n \leq N} \frac{1}{n} \sum_{d \mid n} \mu\left(\frac{n}{d}\right) F(d) \\
& =\sum_{n=1}^{7} \frac{1}{n} \sum_{d \mid n} \mu\left(\frac{n}{d}\right) F(d)+\sum_{n=8}^{N} \frac{1}{n} \sum_{d \mid n} \mu\left(\frac{n}{d}\right) F(d) \geq \pi(7)+\frac{c_{1}}{2} \frac{A^{N}}{N} .
\end{aligned}
$$

This, together with (16), proves the theorem. 
Theorem 3.3 (Mertens orbit theorem) Let $\sigma: \mathrm{D}_{M} \rightarrow \mathrm{D}_{M}$ be the Dyck shift and $\mathcal{M}(N)=$ $\sum_{n \leq N} \frac{O_{\sigma}(n)}{e^{h n}}$. Then there exist constants $C_{3}$ and $C_{4}$ such that

$$
C_{3} \log N \leq \sum_{n \leq N} \frac{O_{\sigma}(n)}{e^{h n}} \leq C_{4} \log N
$$

Proof The dynamical Mertens theorem asserts that

$$
\begin{aligned}
\sum_{n \leq N} \frac{O_{\sigma}(n)}{e^{h n}} & =\sum_{n \leq N} \frac{1}{n e^{h n}} \sum_{d \mid n} \mu\left(\frac{n}{d}\right) F(d) \\
& =\sum_{n \leq N} \frac{1}{n e^{h n}} \sum_{d \mid n, d<n} \mu\left(\frac{n}{d}\right) F(d)+\sum_{n \leq N} \frac{F(n)}{n e^{h n}}
\end{aligned}
$$

First of all, we want to estimate the term

$$
\sum_{n \leq N} \frac{1}{n e^{h n}} \sum_{d \mid n, d<n} \mu\left(\frac{n}{d}\right) F(d) .
$$

Note that

$$
|\mu(n)| \leq 1 \quad \text { and } \quad \sum_{d \mid n, d<n} F(d) \leq\left[\frac{n}{2}\right] F\left(\left[\frac{n}{2}\right]\right)
$$

for all natural numbers $n$. Hence by using Lemma 3.1 we get

$$
\begin{aligned}
\left|\sum_{n=1}^{N} \frac{1}{n e^{h n}} \sum_{d \mid n, d<n} \mu\left(\frac{n}{d}\right) F(d)\right| & \leq \sum_{n=1}^{N} \frac{1}{n e^{h n}} \cdot\left[\frac{n}{2}\right] F\left(\left[\frac{n}{2}\right]\right) \leq c_{7} \sum_{n=1}^{N} \frac{F\left(\left[\frac{N}{2}\right]\right)}{e^{h n}} \\
& \leq c_{7} c_{2} \sum_{n=1}^{N} \frac{A^{\left[\frac{n}{2}\right]}}{A^{n}}=c_{7} c_{2} \sum_{n=1}^{N} \frac{1}{A^{\left[\frac{n}{2}\right]}}=c_{8},
\end{aligned}
$$

since $e^{h n}=A^{n}$. Now

$$
\sum_{n \leq N} \frac{F(n)}{n e^{h n}} \leq c_{2} \sum_{n \leq N} \frac{A^{n}}{n A^{n}}=c_{2}\left(\log N+C+O\left(N^{-1}\right)\right) .
$$

From (18) and (19), we can conclude that

$$
\sum_{n \leq N} \frac{O_{\sigma}(n)}{e^{h n}} \leq c_{9} \log N
$$

For the lower bound, using again (17) and Lemma 3.1 we obtain

$$
\begin{aligned}
\sum_{n \leq N} \frac{O_{\sigma}(n)}{e^{h n}} & =\sum_{n \leq N} \frac{1}{n e^{h n}} \sum_{d \mid n} \mu\left(\frac{n}{d}\right) F(d) \\
& =\sum_{n=1}^{7} \frac{1}{n e^{h n}} \sum_{d \mid n} \mu\left(\frac{n}{d}\right) F(d)+\sum_{n=8}^{N} \frac{1}{n e^{h n}} \sum_{d \mid n} \mu\left(\frac{n}{d}\right) F(d)
\end{aligned}
$$




$$
\begin{aligned}
& \geq \mathcal{M}(7)+\frac{c_{1}}{2} \sum_{n=8}^{N} \frac{A^{n}}{n A^{n}}=\mathcal{M}(7)+\frac{c_{1}}{2} \sum_{n=8}^{N} \frac{1}{n} \\
& \geq c_{10} \log N .
\end{aligned}
$$

This and (20) complete the proof.

Theorem 3.4 (Meissel's orbit theorem) Let $\sigma: \mathrm{D}_{M} \rightarrow \mathrm{D}_{M}$ be the Dyck shift. Then there exist constants $C_{5}$ and $C_{6}$ such that

$$
\frac{C_{5}}{k} \leq \sum_{n=1}^{\infty} \frac{O_{\sigma}(n)}{n^{k} e^{h n}} \leq \frac{C_{6}}{k}
$$

for any $k>0$.

Proof To prove this theorem we first estimate $O(n)$. By using Lemma 3.1, we get

$$
\begin{aligned}
O(n) & =\frac{1}{n} \sum_{d \mid n} \mu\left(\frac{n}{d}\right) F(d) \leq \frac{1}{n} \sum_{d=1}^{n} F(d) \\
& \leq c_{2} \frac{1}{n} \sum_{d=1}^{n} A^{d}=\frac{c_{2} A}{A-1}\left(\frac{A^{n}}{n}\right) .
\end{aligned}
$$

On the other hand, using equation (17), we obtain

$$
O(n)=\frac{1}{n} \sum_{d \mid n} \mu\left(\frac{n}{d}\right) F(d) \geq \frac{c_{2}}{2}\left(\frac{A^{n}}{n}\right) \quad \text { for } n \geq 8
$$

Equations (21) and (22) imply

$$
\sum_{n=1}^{\infty} \frac{O_{\sigma}(n)}{n^{k} e^{h n}} \leq \frac{c_{2} A}{A-1} \sum_{n=1}^{\infty} \frac{A^{n}}{n^{k+1} e^{h n}}=\frac{c_{2} A}{A-1} \sum_{n=1}^{\infty} \frac{1}{n^{k+1}}
$$

since $e^{h n}=A^{n}$. Also

$$
\sum_{n=1}^{\infty} \frac{O_{\sigma}(n)}{n^{k} e^{h n}} \geq \sum_{n=1}^{7} \frac{O_{\sigma}(n)}{n^{k} e^{h n}}+\frac{c_{1}}{2} \sum_{n=8}^{\infty} \frac{1}{n^{k+1}}
$$

To finish this, we use the following inequality:

$$
\int_{1}^{\infty} \frac{d x}{x^{k+1}} \leq \sum_{n=1}^{\infty} \frac{1}{n^{k+1}} \leq 1+\int_{1}^{\infty} \frac{d x}{x^{k+1}}
$$

Calculating the last integral and applying into (23) and (24) gives

$$
\frac{C_{5}}{k} \leq \sum_{n=1}^{\infty} \frac{O_{\sigma}(n)}{n^{k} e^{h n}} \leq \frac{C_{6}}{k} .
$$


A simple formula for the zeta function of the Dyck shift $\mathrm{D}_{M}$ over $2 M$ symbols has been obtained by [17]. He showed that

$$
\zeta_{\sigma}(z)=\frac{2\left(1+\sqrt{1-4 M z^{2}}\right)}{\left(1-2 M z+\sqrt{1-4 M z^{2}}\right)^{2}} .
$$

Keller proved this formula using the so-called circular codes. By circular codes we mean a set $C$ of finite sequences over a finite alphabet $\mathcal{A}$ such that each two-sided infinite periodic sequence of letters from $\mathcal{A}$ has at most one decomposition into words from $C$.

Theorem 3.5 (Dirichlet series) There exist constants $C_{7}$ and $C_{8}$ such that

$$
\frac{C_{7}}{\zeta_{\sigma}(z+1)} \sum_{n=1}^{\infty} \frac{A^{n}}{n^{z+1}} \leq \sum_{n=1}^{\infty} \frac{O_{\sigma}(n)}{n^{z}} \leq \frac{C_{8}}{\zeta_{\sigma}(z+1)} \sum_{n=1}^{\infty} \frac{A^{n}}{n^{z+1}}
$$

where $A=M+1$ and $\zeta(z)$ is the zeta function of the Dyck shift.

Proof By Lemma 3.1, we get

$$
\begin{aligned}
d(z) & =\frac{1}{\zeta(z+1)} \sum_{n=1}^{\infty} \frac{F_{\sigma}(n)}{n^{z+1}} \\
& \leq c_{2} \frac{1}{\zeta(z+1)}\left(A+\frac{A^{2}}{2^{z+1}}+\frac{A^{3}}{3^{z+1}}+\cdots\right) .
\end{aligned}
$$

Also

$$
\begin{aligned}
d(z) & =\frac{1}{\zeta(z+1)} \sum_{n=1}^{\infty} \frac{F_{\sigma}(n)}{n^{z+1}} \\
& \geq c_{1} \frac{1}{\zeta(z+1)}\left(A+\frac{A^{2}}{2^{z+1}}+\frac{A^{3}}{3^{z+1}}+\cdots\right) .
\end{aligned}
$$

\section{Competing interests}

The authors declare that they have no competing interests.

\section{Authors' contributions}

All authors contributed equally to this work. All authors read and approved the final manuscript.

\section{Author details}

'School of Mathematical Sciences, Faculty of Science and Technology, Universiti Kebangsaan Malaysia, Bangi, 43600, Malaysia. ${ }^{2}$ Department of Mathematics and Statistics, School of Quantitative Sciences, UUM CAS, Sintok, Kedah 06010, Malaysia.

\section{Acknowledgements}

The authors would like to acknowledge the grant: UKM Grant DIP-2014-034 and Ministry of Education, Malaysia grant FRGS/1/2014/ST06/UKM/01/1, DIP-2012-31, AP-2013-009, and Modal Insan Berpusat (RAE3) for financial support. The authors also would like to thank the referee for his/her careful reading of the paper and useful suggestions.

Received: 6 May 2015 Accepted: 17 November 2015 Published online: 02 December 2015

\section{References}

1. Lindqvist, P, Peetre, J: On a number theoretic sum considered by Meissel. Nieuw Arch. Wiskd. 15, 175-179 (1997)

2. Patterson, SJ: An Introduction to the Theory of the Riemann Zeta-Function. Cambridge Studies in Advanced Mathematics, vol. 14. Cambridge University Press, Cambridge (1988)

3. Hardy, GH, Wright, EM: An Introduction to the Theory of Numbers. Oxford University Press, Oxford (1938)

4. Artin, M, Mazur, B: On periodic points. Ann. Math. 81(2), 82-99 (1965) 
5. Parry, W: An analogue of the prime number theorem for closed orbits of shifts of finite type and their suspensions. Isr. J. Math. 45, 41-52 (1983)

6. Parry, W, Pollicott, M: An analogue of the prime number theorem for closed orbits of Axiom A flows. Ann. Math. 118, 573-591 (1983)

7. Noorani, MSM: Counting closed orbits of hyperbolic diffeomorphisms. Results Math. 50, 241-257 (2007)

8. Sharp, R: An analogue of Mertens' theorem for closed orbits of Axiom A flows. Bol. Soc. Bras. Mat. 21, 205-229 (1991)

9. Everest, D, Miles, R, Stevens, S, Ward, T: Orbit-counting in non-hyperbolic dynamical systems. J. Reine Angew. Math. 608, 155-182 (2007)

10. Noorani, MSM: Mertens' theorem and closed orbits of ergodic toral automorphisms. Bull. Malays. Math. Sci. Soc. (2) 22, 127-133 (1999)

11. Waddington, S: The prime orbit theorem for quasihyperbolic toral automorphisms. Monatshefte Math. 112, 235-248 (1991)

12. Jaidee, S, Stevens, S, Ward, T: Mertens' theorem for toral automorphisms. Proc. Am. Math. Soc. 139, 1819-1824 (2011)

13. Alsharari, F, Noorani, MSM, Akhadkulov, H: Counting closed orbits for the Dyck shift. Abstr. Appl. Anal. 2014, Article ID 304798 (2014)

14. Marcus, B, Lind, D: An Introduction to Symbolic Dynamics and Coding. Cambridge University Press, Cambridge (1995)

15. Krieger, W: On the uniqueness of equilibrium state. Math. Syst. Theory 8, 97-104 (1974)

16. Hamachi, T, Inoue, K: Embedding shifts of finite type into the Dyck shift. Monatshefte Math. 145, 107-129 (2005)

17. Gerhard, K: Circular codes, loop counting, and zeta-functions. J. Comb. Theory 56, 75-83 (1991)

\section{Submit your manuscript to a SpringerOpen ${ }^{\ominus}$ journal and benefit from:}

- Convenient online submission

Rigorous peer review

- Immediate publication on acceptance

- Open access: articles freely available online

- High visibility within the field

- Retaining the copyright to your article 